\title{
Predicting Readmission Risk Following Coronary Artery Bypass Surgery at the Time of Admission
}

\author{
Zaher Fanari, M.D ${ }^{1,2}$, Daniel Elliott, M.D, M.S.C.E ${ }^{3,4}$, Carla A Russo, M.S ${ }^{4}$, Paul Kolm, Ph.D. \\ ${ }^{4}$, and William S. Weintraub, M.D. ${ }^{1,4}$ \\ ${ }^{1}$ Section of Cardiology, Christiana Care Health System, Newark, DE \\ ${ }^{2}$ Prairie Heart Institute, Springfield, IL \\ ${ }^{3}$ Department of Medicine, Christiana Care Health System, Newark, DE \\ ${ }^{4}$ Value Institute, Christiana Care Health System, Newark, DE
}

\section{Abstract}

Background-Reducing readmissions following hospitalization is a national priority. Identifying patients at high risk for readmission after coronary artery bypass graft surgery (CABG) early in a hospitalization would enable hospitals to enhance discharge planning.

\begin{abstract}
Methods-We developed different models to predict 30-day inpatient readmission to our institution in patients who underwent CABG between January 2010 and April 2013. These models used data available: 1) at admission, 2) at discharge 3) from STS Registry data. We used logistic regression and assessed the discrimination of each model using the c-index. The models were validated with testing on a different patient cohort who underwent CABG between May 2013 and September 2015. Our cohort included 1277 CABG patients: 1,159 in the derivation cohort and 1,018 in the validation cohort.
\end{abstract}

Results-The discriminative ability of the admission model was reasonable (C-index of 0.673). The c-indices for the discharge and STS models were slightly better. (C-index of 0.700 and 0.714 respectively). Internal validation of the models showed a reasonable discriminative admission model with slight improvement with adding discharge and registry data (C-index of 0.641, 0.659 and 0.670 respectively). Similarly validation of the models on the validation cohort showed similar results (C-index of $0.573,0.605$ and 0.595 respectively).

Conclusions-Risk prediction models based on data available early on admission are predictive for readmission risk. Adding registry data did not improved the performance of these models. These simplified models may be sufficient to identify patients at highest risk of readmission following coronary revascularization early in the hospitalization.

\section{Keywords}

Readmission; Risk Prediction; Coronary artery bypass graft surgery

Corresponding Author: Zaher Fanari, M.D., Section of Cardiology, Christiana Care Health System, Prairie Heart Institute, Springfield, IL, 619 E Mason St, Springfield, IL 62769. Tel: +1 (314) 808-1610 Fax: +1 (913) 588-6010, zfanari@ gmail.com.

Disclosure:

None of the authors has any conflict of interest to disclose. 


\section{Introduction}

Coronary artery bypass graft (CABG) surgery is one of the most expensive procedures, with mean charges of nearly $\$ 45,358$ for the index admission. (1) CABG is known to be associated with reasonably high short-term readmission rates. (2) Hospital Readmissions in the US are associated with significant increase in cost and are responsible for an estimate of \$26 billion to the Medicare program alone. (3) The Patient Protection and Affordable Care Act linked many quality outcomes including 30-day readmission rates to hospital reimbursement. (4) The Center for Medicare and Medicaid Services (CMS) publicly reports hospital level 30-day readmission rates for congestive heart failure (CHF), acute myocardial infarction (AMI), and for patients undergoing percutaneous coronary intervention (PCI) with expectation that readmission rates after CABG may be reported in the future. (5-7)

The key for reducing readmission rates depends on delivering high quality care in the inpatient setting and improving the transitional care upon discharge. Given the limitation in resources, early identification of patients at risk for readmission after CABG becomes crucial to direct potential interventions that may help in reducing readmission and improving hospital quality of care. Although many studies have identified strong predictors for readmissions risk for $\mathrm{CABG}(2,8)$, to date there are only two risk models specifically designed to predict readmission risk for patients undergoing CABG.(9-11) However these risk models used registry data at the time of discharge to identify patients at high risk for readmission. These risk models did not address the possibility of using data available prior to or early after admission to create a model that may be helpful in identifying those at risk early in an admission.

We sought to develop and validate a risk model to predict readmission risk after CABG using available clinical and administrative data available within our hospital system at the time of admission, and to determine the incremental benefit to risk assessment of adding 1) clinical information available at the time of discharge, and 2) registry data from the STS (Society Of Thoracic Surgeons) Registry. Our findings will inform the use of clinical data within hospital systems to prospectively risk-stratify patients to support the cost-effective application of care management or other resources with the intent to reduce readmission.

\section{Methods}

\section{Study Design, Population, and Setting}

We conducted a retrospective cohort of all patients with revascularization with CABG at Christiana Care Health System between January 1, 2010 and April 1, 2015. Christiana Care is a large system that comprises two hospitals with more than 1,100 beds as well as a variety of outpatient and other services in facilities and provides the majority of cardiovascular care in Delaware and the surrounding area with an estimate of 1700 PCI and more than 600 open heart surgery annually. We identified all patients who were discharged alive following CABG. We further divided the cohort into 1) a derivation cohort that was used to develop the 3 separate prediction models and included CABG patients admitted between April 1, 2010 and April 30, 2013; and 2) a validation cohort that was used to test the prediction 
models and included those admitted between May 1, 2013 and September 30, 2015.

Importantly, the validation cohort included patients that were included in a longitudinal care management program for patients following coronary revascularization. Patients were enrolled in the program during the hospitalization and followed with telephonic care management following discharge. The Christiana Care Institutional Review Board approved the study.

\section{Outcomes}

We identified inpatient, non-elective readmissions to Christiana Care within 30 days of discharge from the index procedure. We identified readmissions at our own system and we used QualityNet Data from CMS to identify readmissions at other hospitals.

\section{Candidate Variables and Model Derivation}

Candidate variables for the prediction model were drawn from three sources: 1)

administrative and billing data from the data warehouse at Christiana Care (demographics, previous utilization, and comorbidities; 2) clinical data including initial and discharge laboratory and vital signs from key clinical systems; and 3) registry information from the STS Registry for data concerning anatomic and procedural information. Comorbidities were classified from administrative data using the Elixhauser classification. (12)

In order to determine the incremental value of additional information gathered across the hospital visit, we developed three models that sequentially added information available during the hospitalization: 1) an admission model that included only variables available at the time the patient arrived at the hospital, 2) a discharge model that included administrative and clinical information available at the time of discharge; and 3) a discharge model that also included anatomic and procedural information from the STS Registry. This progression of models was chosen based on the timing of availability of this data in the clinical setting. STS registry information, for example, is collected by staff following discharge and is not available for operational purposes at the time of discharge. These variables and the progress in building each model are shown in Table 1.

Hierarchical logistic regression was used to model readmissions (a patient may have had more than one). Derivation models were developed by a combination of forward selection and backward elimination of variables. Variables were entered if $p \leq .2$ and removed if $p>$. 2. Reduced models were compared to larger models by likelihood ratio tests. Fractional polynomial (FP) regression was used to assess non-linearity of continuous variables. Cubic splines were then used to determine categories for nonlinear continuous variables. Although adding variables to the sequential models will likely change the estimation of odds ratios (as well as contribution to the predictive ability of the model), variables were retained in subsequent models regardless of their contribution to predictive ability. Model discrimination was assessed by the c-statistic and model calibration was assessed by plotting observed readmission rates with deciles of model-predicted rates.

Models were developed for CABG patients admitted to the hospital between April 1, 2010 and April 30, 2013. Internal model validity was assessed by bootstrap methods -500 bootstrap replicates with replacement were drawn to calculate bias-corrected c-indices. The 
derivation models were then applied to patients admitted between May 1, 2013 and September 30, 2015 to assess external validity.

\section{Results}

The total number of CABG patients was 1,277 including 1,159 in the derivation cohort and 1,018 in the validation cohort. These patients had a total of 2,183 hospitalizations; 1,163 in the derivation cohort and 1,020 in the validation cohort. The readmission rate was $14.2 \%$. Table-2 shows the demographic and clinical characteristics of the derivation and validation cohorts.

The admission, admission + discharge and admission + discharge + STS registry models are presented in Tables 3-5 respectively. Increasing age, female gender, non-white race, previous hospitalization within 6 months of the current hospitalization, previous AMI and previous PCI were risk factors for readmission, whereas private insurance carrier, self-payment of hospitalization, and elective procedure were predictive for lower risk of readmission. The cindex for the admission model was $0.673(95 \% \mathrm{CI}=0.625-0.720)$. The addition of variables available at discharge, CHF, diabetes, depression, length of hospital stay, discharged to home with services and discharged to other facilities resulted in a marginal increase ( $\mathrm{p}=0.063)$ in the $\mathrm{c}$-index to $0.700(95 \% \mathrm{CI}=0.654-0.747)$. Addition of STS registry variables, $\mathrm{LVEF}<50 \%$, coumadin administered post-op and lipid-lowering meds administered post-op, marginally improved the c-index to 0.714 (95\% CI $=0.669-0.758, \mathrm{p}$ $=0.104)$.

Internal validation of the admission, admission + discharge and admission + discharge + registry models resulted in c-indices of $0.641,0.659$ and 0.670 respectively. External validation of the admission, admission + discharge and admission + discharge + registry models resulted in c-indices of $0.573,0.605$ and 0.595 respectively. Figure 1 A-C shows calibration plots for each of the models for the derivation and the validation cohorts.

\section{Discussion}

We developed 3 models, one at admission and two at discharge. The early risk prediction model was reasonably predictive for readmission risk and adding data available at discharge only minimally improved the predictive performance of the model. Our findings suggest that a simple model comprised of data available at hospital admission may be sufficient to identify CABG patients at highest risk of readmission. Such a model may be used to help in optimizing care and reducing subsequent readmissions. Hospitals will be able to direct case management personnel to better coordinate more robust counseling about discharge planning, new medications, potential expectations for post discharge care at home, arranging for home health or referral rehabilitation services at home or at rehabilitation facilities, setting a closer and more frequent outpatient follow-up. Case management teams have been shown to be an effective strategy for reducing readmissions and associated costs especially if it was directed towards those who are at higher risk for readmission. $(13,14)$

There is an increased need for reducing readmission rates nationwide, especially with pressure from the Affordable Care Act with financial incentives for hospitals to minimize 
readmissions.(3) Two studies based on the New York state registry identified risk factors associated with CABG readmission early in 2003 and in an updated version in 2011. (2,8) In the updated report, the most common reasons for readmission were post-operative infection (16.9\%), heart failure (12.8\%), and "other complications of surgical and medical care" $(9.8 \%)$. Risk factors associated with increased risk of readmissions were increasing age, female sex, African-American race, higher body mass index, numerous comorbidities, 2 post-operative complications (renal failure and unplanned cardiac reoperation), Medicare or Medicaid insurance, discharges to a skilled nursing facility, saphenous vein grafts, and longer initial lengths of stay. Another report showed that in admission to the risk factors reported by the New York registry, abnormal discharge serum creatinine was associated with increased readmission risk. (15) Similarly, data from California CABG clinical registry showed that although the rate of readmissions after $\mathrm{CABG}$ varied widely among different hospitals, patient demographic and clinical risk factors were more predictive for readmission than hospital characteristics. The patients' factors predictive of readmission included age, sex, and lower zip code-level median household income. (16)

Risk predictions model were developed to predict readmission rate in the general hospital population with LACE index score and HOSPITAL Score. Both models had fair discriminative ability with $\mathrm{C}$ - statistics of 0.68 and 0.71 respectively, but they used model data available only at discharge, so it does not offer the opportunity to identify high-risk patients for early interventions to reduce the risk of readmissions. $(17,18)$ Multiple models were developed to predict readmission in heart failure patients and after acute myocardial infarction. (19-21) But such models risk stratify a higher risk population, use many candidate variables that are available only at discharge, and may not be applicable in predicting risk for readmission after CABG. (21)

There are three risk models that were developed to predict readmission after CABG. (9-11) The first model is derived from the Israeli CABG Study (ISCAB) of 4,835 patients with isolated CABG in Israel in 1994. (9) This logistic model used data on preoperative comorbidities, operative factors, immediate post-operative complications and sociodemographic characteristics as well as provider characteristics was developed. The model had a reasonable predictive C-statistics of 0.65 . The second model was derived from the STS database in 11 hospitals. The data used in this model are mainly clinical data. (10) The model had a reasonable C-index of 0.643 and similarly to our model when it was validated on a different patients' cohort, the c-index dropped significantly to 0.57 . The third model was developed from linking STS registry data to CMS claims and included 162572 CABG admissions. The $\mathrm{C}$ - statistics to this model was also reasonable with 0.648 .

Although these models showed that clinical risk factors could be predictive of CABG readmission, they also had some limitations. First, none of them added previous utilization as an important potential predictor of readmission. Second, they all used registry data that would need abstracting and that may be subjective to inaccurate coding and/or not inclusive of all co-morbidities and complications that may impact and predict readmission. Finally, the models included some variables that are not available at the time of the admission (i.e. in-hospital use of intra aortic balloon bump and blood product...etc). 
In comparison to the previously available models, our risk models have multiple advantages. First, our admission model provides the chance to predict the risk of readmissions at the moment of admission even before any available vitals or labs, and this early recognition helps in planning the admission and potential interventions to those at risk as early as possible to prevent readmissions. Second; our models showed that general risk factors including demographic data and previous utilization may be the strongest determinant of readmission risk, Third, the admission model used readily available hospital/outpatient-level clinical and demographic data that can be easily obtained electronically by preset tools without the need for chart review and abstracting. Finally, our results showed that adding registry data with complicated information about complications/anatomy was not additive to more general measures of comorbidity and risk.

There are multiple limitations to our work. First, data are derived from a single center and may not represent patient population nationwide. Our system provides the overwhelming majority of care for Delaware State including urban, suburban and rural areas. Therefore, our findings and observed patterns of care may not be completely generalizable to other hospitals settings, particularly those with lower degrees of market capture. Second, while our models had reasonable discriminative ability to predict high-risk patients for readmission, our models are still not able to discriminate preventable form non-preventable readmissions. Third, although we tried to make the models parsimonious, as readmission risk is multifactorial and not dependent on a small number of strong predictors, the models still include a large number of variables $(14,21$ and 25$)$ to retain sufficient predictive ability. However, we believe that both web based and other information technologies tools can ease their implementation in routine clinical care. Also our admission model uses a readily available data at admission to predict those at risk when it is pertinent and useful. Fourth, Our models are still not optimally disseminative as the C- statistics is still less than 0.80 . (22) However, that was the case for all readmission models evaluated in the literature up till now, which may suggest that it is still very challenging to predict the readmission process using any clinical or administrative data. Finally and importantly, the model performed poorly in the validation cohort, but this may reflect the impact on utilization of a longitudinal care management program during that period. Subsequent studies should seek to validate our model and/or the approach using clinical data in populations that are not simultaneously receiving an intervention designed to impact utilization.

Despite these limitations, this study represents novel work to build a risk model that provides sufficient discrimination to identify those at risk for readmission after CABG, and may help in guiding better resources including case management teams to those at risk, and subsequently help in reducing readmission and cost.

\section{Conclusion}

We demonstrated that a model based on clinical data available at the time of admission has sufficient discrimination and calibration to predict those at risk for readmission after CABG and is only marginally improved by adding more complete clinical data. Adding administrative, and registry data on top of already available clinical data did not increase predictively of readmission risk. 
Models such as this one could allow providers and health systems to target high-risk patients with enhanced discharge planning during the course of the hospitalization, and this may improve the ability to avoid readmissions in a cost-effective manner. Future work should explore the validation of this approach in other settings.

\title{
Acknowledgments
}

\author{
Funding:
}

Supported by grant number 1C1CMS331027 from the Department of Health and Human Services, Centers for Medicare \& Medicaid Services (PI: Weintraub) and by an Institutional Development Award (IDeA) from the National Institute of General Medical Sciences of the National Institutes of Health grant number U54-GM104941 (PI: Binder-Macleod). The contents of this publication are solely the responsibility of the authors and do not necessarily represent the official views of the U.S. Department of Health and Human Services or any of its agencies. The research presented here was conducted by the awardee. Findings might or might not be consistent with or confirmed by the findings of the independent evaluation contractor.

\section{References}

1. Birkmeyer JD, Gust C, Baser O, Dimick JB, Sutherland JM, Skinner JS. Medicare payments for common inpatient procedures: implications for episode-based payment bundling. Health services research. 2010; 45:1783-95. [PubMed: 20698899]

2. Hannan EL, Racz MJ, Walford G, et al. Predictors of readmission for complications of coronary artery bypass graft surgery. JAMA : the journal of the American Medical Association. 2003; 290:773-80. [PubMed: 12915430]

3. Wasfy JH, Rosenfield K, Zelevinsky K, et al. A prediction model to identify patients at high risk for 30-day readmission after percutaneous coronary intervention. Circulation Cardiovascular quality and outcomes. 2013; 6:429-35. [PubMed: 23819957]

4. Pepi M, Evangelista A, Nihoyannopoulos P, et al. Recommendations for echocardiography use in the diagnosis and management of cardiac sources of embolism: European Association of Echocardiography (EAE) (a registered branch of the ESC). European journal of echocardiography : the journal of the Working Group on Echocardiography of the European Society of Cardiology. 2010; 11:461-76.

5. Krumholz HM, Normand SL, Spertus JA, Shahian DM, Bradley EH. Measuring performance for treating heart attacks and heart failure: the case for outcomes measurement. Health affairs. 2007; 26:75-85. [PubMed: 17211016]

6. Krumholz HM, Normand SL. Public reporting of 30-day mortality for patients hospitalized with acute myocardial infarction and heart failure. Circulation. 2008; 118:1394-7. [PubMed: 18725492]

7. Kereiakes DJ. Return to sender hospital readmission after percutaneous coronary intervention. Journal of the American College of Cardiology. 2009; 54:908-10. [PubMed: 19712800]

8. Hannan EL, Zhong Y, Lahey SJ, et al. 30-day readmissions after coronary artery bypass graft surgery in New York State. JACC Cardiovascular interventions. 2011; 4:569-76. [PubMed: 21596331]

9. Zitser-Gurevich Y, Simchen E, Galai N, Braun D. Prediction of readmissions after CABG using detailed follow-up data: the Israeli CABG Study (ISCAB). Medical care. 1999; 37:625-36. [PubMed: 10424633]

10. Lancey R, Kurlansky P, Argenziano M, et al. Uniform standards do not apply to readmission following coronary artery bypass surgery: a multi-institutional study. The Journal of thoracic and cardiovascular surgery. 2015; 149:850-7 e1. discussion 857. [PubMed: 25293357]

11. Shahian DM, He X, O’Brien SM, et al. Development of a clinical registry-based 30-day readmission measure for coronary artery bypass grafting surgery. Circulation. 2014; 130:399-409. [PubMed: 24916208]

12. Southern DA, Quan H, Ghali WA. Comparison of the Elixhauser and Charlson/Deyo methods of comorbidity measurement in administrative data. Medical care. 2004; 42:355-60. [PubMed: 15076812] 
13. Kolbasovsky A, Zeitlin J, Gillespie W. Impact of point-of-care case management on readmissions and costs. The American journal of managed care. 2012; 18:e300-6. [PubMed: 22928799]

14. Gilbert P, Rutland M, Brockopp D. Redesigning the work of case management: testing a predictive model for readmission. Am J Manag Care. 2013 Nov 19.(10 Spec No):eS19-eSP25. [PubMed: 24511885]

15. Price JD, Romeiser JL, Gnerre JM, Shroyer AL, Rosengart TK. Risk analysis for readmission after coronary artery bypass surgery: developing a strategy to reduce readmissions. Journal of the American College of Surgeons. 2013; 216:412-9. [PubMed: 23313544]

16. Li Z, Armstrong EJ, Parker JP, Danielsen B, Romano PS. Hospital variation in readmission after coronary artery bypass surgery in California. Circulation Cardiovascular quality and outcomes. 2012; 5:729-37. [PubMed: 22949489]

17. van Walraven C, Dhalla IA, Bell C, et al. Derivation and validation of an index to predict early death or unplanned readmission after discharge from hospital to the community. CMAJ : Canadian Medical Association journal = journal de l'Association medicale canadienne. 2010; 182:551-7.

18. Donze J, Aujesky D, Williams D, Schnipper JL. Potentially avoidable 30-day hospital readmissions in medical patients: derivation and validation of a prediction model. JAMA internal medicine. 2013; 173:632-8. [PubMed: 23529115]

19. Philbin EF, DiSalvo TG. Prediction of hospital readmission for heart failure: development of a simple risk score based on administrative data. Journal of the American College of Cardiology. 1999; 33:1560-6. [PubMed: 10334424]

20. Yamokoski LM, Hasselblad V, Moser DK, et al. Prediction of rehospitalization and death in severe heart failure by physicians and nurses of the ESCAPE trial. Journal of cardiac failure. 2007; 13:813. [PubMed: 17338997]

21. Krumholz HM, Lin Z, Drye EE, et al. An administrative claims measure suitable for profiling hospital performance based on 30-day all-cause readmission rates among patients with acute myocardial infarction. Circulation Cardiovascular quality and outcomes. 2011; 4:243-52. [PubMed: 21406673]

22. Manitoba Centre for Health Policy. Term: C-statistic. 2011. Retrieved September 7, 2016, from http://mchp-appserv.cpe.umanitoba.ca/viewDefinition.php?definitionID=104234 

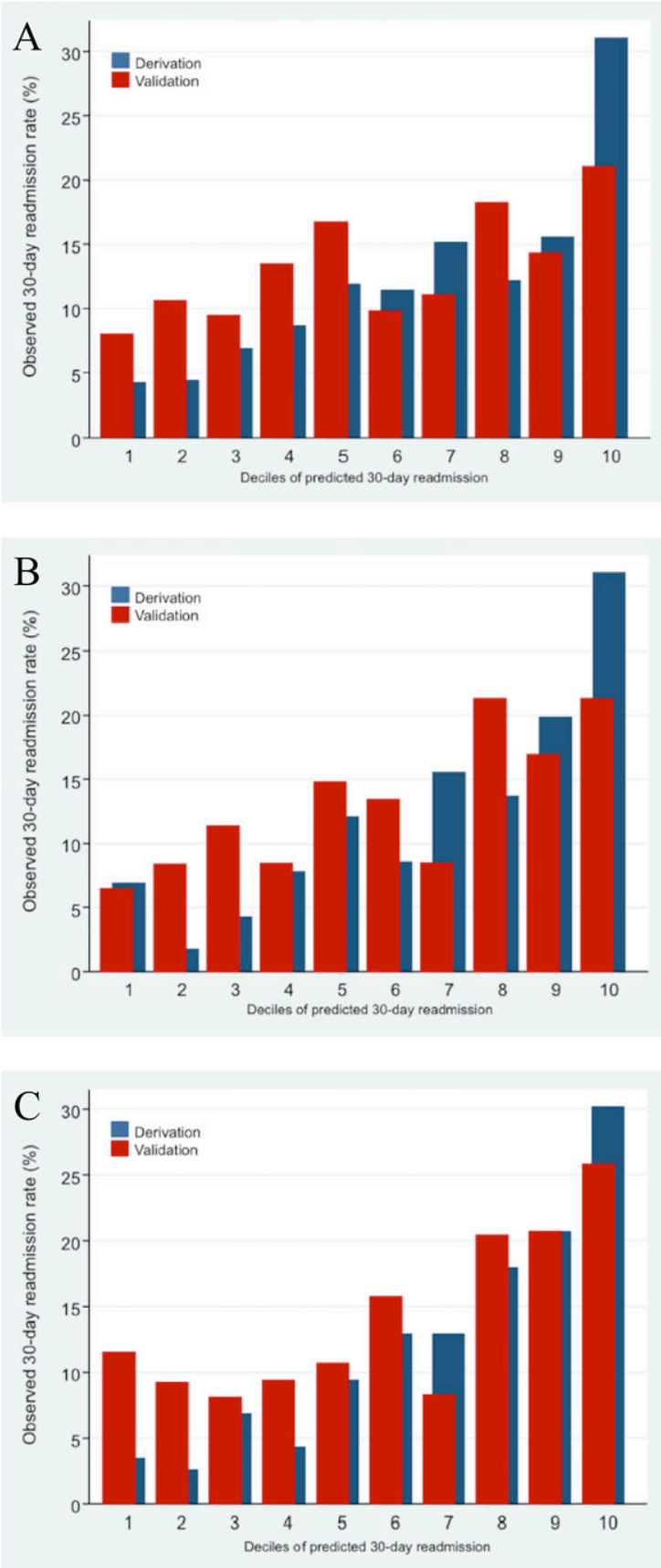

Figure 1.

A- Calibration Plot Of Admission Model For CABG And Application To Test Data.

B- Calibration Plots For Admission + Discharge Model For CABG And Application To Test Data.

C- Calibration Plots For Admission + Discharge + Registry Model For CABG And Application To Test Data. 
Table 1

Variables by Data class and Sequential model development.

\begin{tabular}{|c|c|c|}
\hline \multicolumn{3}{|l|}{ Variables by Data class } \\
\hline Baseline / admission & Discharge & STS \\
\hline Age & Length of stay & Post-op complication \\
\hline Sex & AMI indication & LVEF \\
\hline Race & Any ICU stay & ACE/ARB post-op \\
\hline Insurance & Weekend discharge & Beta Blocker post-op \\
\hline Elective status & $\begin{array}{l}\text { Discharge Location } \\
\text { Home } \\
\text { Home With Services } \\
\text { Skilled Nursing Facility } \\
\text { Others Facility }\end{array}$ & ADP post-op \\
\hline Previous AMI & \multirow{7}{*}{$\begin{array}{l}\begin{array}{l}\text { Updated Elixhauser } \\
\text { comorbidities }\end{array} \\
\cdot \text { Comorbidity count } \\
\cdot \text { CHF } \\
\cdot \text { COPD } \\
\cdot \text { Diabetes } \\
\cdot \text { Renal failure } \\
\cdot \text { Perivascular disease } \\
\text { - Valve disease } \\
\cdot \text { Electrolyte imbalance } \\
\text { - Obesity }\end{array}$} & \multirow{7}{*}{$\begin{array}{l}\text { Antiarrhythmic med post-op } \\
\text { Lipid-lowering med post-op } \\
\text { Statin at discharge } \\
\text { Coumadin post-op } \\
\text { Canadian Classification } \\
\text { System Angina Class }\end{array}$} \\
\hline$\underline{\text { Previous PCI }}$ & & \\
\hline Previous CABG & & \\
\hline Weekend admission & & \\
\hline \multirow[t]{3}{*}{ Previous hospitalization within 6 months } & & \\
\hline & & \\
\hline & & \\
\hline Model & \multicolumn{2}{|l|}{ Data classes included } \\
\hline 1 & \multicolumn{2}{|l|}{ Baseline - admission } \\
\hline 2 & \multicolumn{2}{|c|}{ Baseline - admission / discharge } \\
\hline 3 & \multicolumn{2}{|c|}{ Baseline - admission / discharge / STS Registry } \\
\hline
\end{tabular}

All variables in each set were initially entered into the model and then removed by elimination criteria.

Variables retained in each model were retained in the subsequent model.

Abbreviations:

STS= Society of Thoracic Surgery; PCI= Percutaneous Intervention; AMI= Acute Myocardial Infarction; LVEF= Left Ventricle Ejection Fraction; ICU= Intensive Care Unit; TIMI= Thrombolysis in Myocardial Infarction; ACEI= Angiotensin Converting Enzyme Inhibitor; $\mathrm{ARB}=\mathrm{Angiotensin}$ Receptor Inhibitor; $\mathrm{CABG}=$ Coronary Artery Bypass Grafting; COPD= Chronic Obstructive Pulmonary Disease. NSTEMI= Non-ST Elevation Myocardial Infarction; STEMI= ST Elevation Myocardial Infarction. 
Table 2

CABG Derivation and Validation Cohorts Demographic and Clinical Characteristics.

\begin{tabular}{|c|c|c|c|}
\hline \multicolumn{2}{|l|}{ Variable } & Derivation cohort $N=1,159$ & Validation cohort $N=1,018$ \\
\hline \multicolumn{2}{|l|}{ Age (years) } & $67.0 \pm 11.1$ & $66.8 \pm 10.7$ \\
\hline \multicolumn{2}{|l|}{ Female $(\%)$} & 25.8 & 26.0 \\
\hline \multirow[t]{3}{*}{ Race $(\%)$} & White & 86.7 & 83.4 \\
\hline & Black & 10.0 & 11.5 \\
\hline & Other & 3.3 & 5.1 \\
\hline \multirow[t]{5}{*}{ Insurance $(\%)$} & Medicare & 61.1 & 60.8 \\
\hline & Medicaid & 5.2 & 6.5 \\
\hline & Private & 30.0 & 27.9 \\
\hline & Self pay & 2.2 & 0.9 \\
\hline & Other & 1.5 & 3.9 \\
\hline \multicolumn{2}{|l|}{ Elective (\%) } & 49.4 & 39.3 \\
\hline \multicolumn{2}{|l|}{ Transfer $(\%)$} & 5.8 & 6.2 \\
\hline \multicolumn{2}{|l|}{ Previous AMI } & 6.4 & 6.8 \\
\hline \multicolumn{2}{|l|}{ Previous PCI } & 22.2 & 22.1 \\
\hline \multicolumn{2}{|l|}{ Previous CABG } & 1.6 & 1.4 \\
\hline \multirow{3}{*}{$\begin{array}{l}\text { Previous IOE } \\
\text { hospitalization } \\
\text { within } 6 \text { mos. (\%) }\end{array}$} & None & 52.1 & 52.7 \\
\hline & 1 & 34.9 & 33.3 \\
\hline & $>1$ & 13.0 & 13.9 \\
\hline \multirow[t]{5}{*}{ Comorbidities } & Total count & $4.6 \pm 2.8$ & $5.1 \pm 2.9$ \\
\hline & $\mathrm{CHF}(\%)$ & 32.1 & 32.4 \\
\hline & $\operatorname{COPD}(\%)$ & 28.2 & 29.5 \\
\hline & Diabetes $(\%)$ & 44.4 & 47.0 \\
\hline & Renal failure (\%) & 21.1 & 19.5 \\
\hline \multicolumn{2}{|l|}{ LOS (days) } & $8.8 \pm 7.1$ & $8.7 \pm 8.8$ \\
\hline \multirow{4}{*}{$\begin{array}{l}\text { Discharge } \\
\text { disposition (\%) }\end{array}$} & Home & 9.4 & 6.8 \\
\hline & Home w/ services & 72.0 & 70.0 \\
\hline & SNF & 15.3 & 19.0 \\
\hline & Other facility & 3.3 & 4.2 \\
\hline
\end{tabular}

Gender and race percentages are based on number of patients; all other categorical variables on number of hospitalizations.

Age, comorbidity count and LOS are mean \pm 1 standard deviation 
Table 3

Final CABG Admission Model Predicting 30-Day Inpatient Readmission.

\begin{tabular}{|l|l|l|l|l|}
\hline \multicolumn{2}{|l|}{ Variable } & Odds Ratio & 95\% Confidence Interval & P Value \\
\hline \multicolumn{2}{|l|}{ Age (10-Year Increments) } & 1.07 & $0.88-1.31$ & .48 \\
\hline \multirow{2}{*}{ Female } & 1.12 & $0.75-1.68$ & .56 \\
\hline \multirow{2}{*}{ Race } & White & 1.00 & ------------- & -------- \\
\cline { 2 - 5 } & Black & 1.74 & $1.03-2.94$ & .04 \\
\cline { 2 - 5 } & Other Race & 1.09 & $0.35-3.40$ & .88 \\
\hline \multirow{2}{*}{ Insurance } & Private Insurance & 0.57 & $0.34-0.97$ & .04 \\
\cline { 2 - 5 } & Self Pay & 0.21 & $0.03-1.79$ & .15 \\
\hline Weekend Admission & 0.64 & $0.33-1.25$ & .19 \\
\hline Elective & 0.59 & $0.40-0.88$ & .009 \\
\hline Previous Hospitalization Within 6 Months & 1.25 & $1.05-1.50$ & .01 \\
\hline \multicolumn{2}{|l|}{ Previous AMI } & 1.66 & $0.85-3.26$ & .14 \\
\hline \multicolumn{2}{|l|}{ Previous PCI } & 1.73 & $1.10-2.72$ & .02 \\
\hline
\end{tabular}


Table 4

CABG admission + discharge model predicting 30-day inpatient readmission

\begin{tabular}{|c|c|c|c|c|}
\hline \multicolumn{2}{|l|}{ Variable } & Odds Ratio & 95\% Confidence Interval & $P$ Value \\
\hline \multicolumn{2}{|l|}{ Age (10-Year Increments) } & 1.05 & $0.85-1.29$ & .67 \\
\hline \multicolumn{2}{|l|}{ Female } & 0.99 & $0.65-1.50$ & .96 \\
\hline \multirow[t]{3}{*}{ Race } & White & 1.00 & --------------- & ------ \\
\hline & Black & 1.67 & $0.97-2.88$ & .06 \\
\hline & Other Race & 1.08 & $0.34-3.41$ & .89 \\
\hline \multirow[t]{2}{*}{ Insurance } & Private Insurance & 0.60 & $0.35-1.03$ & .06 \\
\hline & Self Pay & 0.27 & $0.03-2.17$ & .22 \\
\hline \multicolumn{2}{|l|}{ Weekend Admission } & 0.60 & $0.30-1.17$ & .14 \\
\hline \multicolumn{2}{|l|}{ Elective } & 0.69 & $0.46-1.04$ & .08 \\
\hline \multicolumn{2}{|l|}{$\begin{array}{l}\text { Previous Hospitalization } \\
\text { Within } 6 \text { Months }\end{array}$} & 1.17 & $0.98-1.40$ & .08 \\
\hline \multicolumn{2}{|l|}{ Previous AMI } & 1.67 & $0.86-3.25$ & .13 \\
\hline \multicolumn{2}{|l|}{ Previous PCI } & 1.68 & $1.07-2.64$ & .24 \\
\hline \multicolumn{2}{|l|}{ Length Of Stay (Days) } & 1.02 & $1.00-1.04$ & .09 \\
\hline \multirow[t]{3}{*}{ Elixhauser Co-Morbidities } & $\mathrm{CHF}$ & 1.33 & $0.90-1.99$ & .15 \\
\hline & Diabetes & 1.32 & $0.90-1.94$ & .16 \\
\hline & Depression & 1.59 & $1.01-2.52$ & .04 \\
\hline \multirow[t]{2}{*}{ Discharge Location } & Home W/ Services & 0.72 & $0.47-1.10$ & .13 \\
\hline & Other Facility & 0.40 & $0.12-1.29$ & .12 \\
\hline
\end{tabular}


Table 5

CABG admission + discharge + registry model predicting 30-day inpatient

\begin{tabular}{|c|c|c|c|c|}
\hline \multicolumn{2}{|l|}{ Variable } & Odds Ratio & 95\% Confidence Interval & P Value \\
\hline \multicolumn{2}{|l|}{ Age (10-Year Increments) } & 1.05 & $0.85-1.31$ & .63 \\
\hline \multicolumn{2}{|l|}{ Female } & 0.99 & $0.65-1.52$ & .98 \\
\hline \multirow[t]{3}{*}{ Race } & White & 1.00 & ---------------- & ------- \\
\hline & Black & 1.69 & $0.98-2.93$ & .06 \\
\hline & Other Race & 1.24 & $0.41-3.77$ & .71 \\
\hline \multirow[t]{2}{*}{ Insurance } & Private Insurance & 0.59 & $0.34-1.02$ & .06 \\
\hline & Self-Pay & 0.26 & $0.03-1.97$ & .19 \\
\hline \multicolumn{2}{|l|}{ Weekend Admission } & 0.59 & $0.30-1.16$ & .13 \\
\hline \multicolumn{2}{|l|}{ Elective } & 0.69 & $0.46-1.04$ & .08 \\
\hline \multicolumn{2}{|c|}{ Previous Hospitalization Within 6 Months } & 1.18 & $0.99-1.41$ & .07 \\
\hline \multicolumn{2}{|l|}{ Previous AMI } & 1.61 & $0.82-3.13$ & .16 \\
\hline \multicolumn{2}{|l|}{ Previous PCI } & 1.67 & $1.06-2.63$ & .02 \\
\hline \multirow[t]{3}{*}{ Elixhauser Co-Morbidities } & $\mathrm{CHF}$ & 1.08 & $0.69-1.69$ & .73 \\
\hline & Diabetes & 1.32 & $0.90-1.94$ & .15 \\
\hline & Depression & 1.65 & $1.04-2.61$ & .03 \\
\hline \multirow[t]{2}{*}{ Discharge Location } & Home W/ Services & 0.73 & $0.48-1.12$ & .14 \\
\hline & Other Facility & 0.44 & $0.14-1.40$ & .16 \\
\hline \multicolumn{2}{|l|}{ Length Of Stay } & 1.02 & $1.00-1.04$ & .08 \\
\hline \multicolumn{2}{|l|}{ LVEF $<50 \%$} & 1.45 & $0.95-2.22$ & .08 \\
\hline \multicolumn{2}{|l|}{ Coumadin Post-Op } & 1.95 & $1.08-3.54$ & .03 \\
\hline \multicolumn{2}{|l|}{ Lipid-Lowering Med Post-Op } & 2.17 & $0.92-5.13$ & .07 \\
\hline
\end{tabular}

